\title{
Four-Year Experience Using an Advanced Interdisciplinary Hybrid Operating Room : Potentials in Treatment of Cere- brovascular Disease
}

Hong Jun Jeon, M.D., Jong Young Lee, M.D., Ph.D.,, Byung-Moon Cho, M.D., Ph.D., Dae Young Yoon, M.D., Ph.D., ${ }^{1}$ Sae-Moon Oh, M.D., Ph.D. ${ }^{3}$

Departments of Neurosurgery, Radiology, Kangdong Sacred Heart Hospital, Hallym University College of Medicine, Seoul, Korea Health Insurance Review and Assessment Service, Seoul, Korea

Objective : To describe our experiences with a fully equipped high-end digital subtraction angiography (DSA) system within a hybrid operating room (OR).

Methods : A single-plane DSA system with 3-dimensional rotational angiography, cone-beam computed tomography (CBCT), and real-time navigation software was used in our hybrid OR. Between April 2014 and January 2018, 191 sessions of cerebrovascular procedures were performed in our hybrid OR. After the retrospective review of all cases, the procedures were categorized into three subcategorical procedures : combined endovascular and surgical procedure, complementary rescue procedure during intervention and surgery, and frameless stereotaxic operation.

Results : Forty-nine of 191 procedures were performed using hybrid techniques. Four cases of blood blister aneurysms and a ruptured posterior inferior cerebellar artery aneurysm were treated using bypass surgery and endovascular trapping. Eight cases of ruptured aneurysm with intracranial hemorrhage $(\mathrm{ICH})$ were treated by partial embolization and surgical clipping. Six cases of ruptured arteriovenous malformation with $\mathrm{ICH}$ were treated by Onyx embolization of nidus and subsequent surgical removal of nidus and ICH. Two (5.4\%) of the 37 cases of pre-mature rupture during clipping were secured by endovascular coil embolization. In one $(0.8 \%)$ complicated case of 103 intra-arterial thrombectomy procedures, emergency surgical embolectomy with bypass surgery was performed. In 27 cases of $\mathrm{ICH}$, frameless stereotaxic hematoma aspiration was performed using XperGuide ${ }^{\circledR}$ system (Philips Medical Systems, Best, the Netherlands). All procedures were performed in single sessions without any procedural complications.

Conclusion : Hybrid OR with a fully equipped DSA system could provide precise and safe treatment strategies for cerebrovascular diseases. Especially, we could suggest a strategy to cope flexibly in complex lesions or unexpected situations in hybrid OR. CBCT with real-time navigation software could augment the usefulness of hybrid OR.

Key Words : Hybrid · Operating rooms · Cone-beam computed tomography · Cerebrovascular disorders.

- Received : October 24, 2018 •Revised : November 5, 2018 •Accepted : November 13, 2018

- Address for reprints : Jong Young Lee, M.D., Ph.D.

Department of Neurosurgery, Kangdong Sacred Heart Hospital, Hallym University College of Medicine, 150 Seongan-ro, Gangdong-gu, Seoul 05355, Korea Tel : +82-2-2224-2236, Fax : +82-2-743-6385, E-mail : nsyjee@gmail.com

This is an Open Access article distributed under the terms of the Creative Commons Attribution Non-Commercial License (http://creativecommons.org/licenses/by-nc/4.0) which permits unrestricted non-commercial use, distribution, and reproduction in any medium, provided the original work is properly cited. 


\section{INTRODUCTION}

The usefulness of a hybrid operating room (OR), which is fully equipped with digital subtraction angiography (DSA), has been demonstrated by neurosurgeons ${ }^{5,6,13-15)}$. In particular, high-resolution three-dimensional rotational angiography (3DRA) was integrated in the OR to improve the intraoperative diagnostic capabilities ${ }^{6}$. Going beyond intraoperative diagnostic control angiography, various types of combined surgical and endovascular procedures have been performed in single sessions in the hybrid $\mathrm{OR}^{5,11,13,15,23)}$. Newly developed real-time navigation software merged with cone-beam computed tomography (СВСТ) can be used in frameless navigation and in access to deep-seated intracranial lesions ${ }^{14}$. Currently, a hybrid OR is becoming popular in studies of various cerebrovascular diseases.

In accordance with these modern trends, we aimed to introduce a fully equipped high-end DSA system in one of our ORs. We present the organizational points, clinical results, advantages and drawbacks from the treatment of patients with cerebrovascular diseases in hybrid OR.

\section{MATERIALS AND METHODS}

This retrospective study was approved by the Institutional Review Board at Kangdong Sacred Heart Hospital and in- formed consent was waived (IRB No. 2018-06-011). Patient and procedural data and clinical records have been prospectively recorded in a database continuously and were retrospectively analyzed.

\section{Patient population}

Between April 2014 and January 2018, 191 sessions of cerebrovascular procedures were performed in our hybrid OR by one neurosurgeon who perform open surgery and two hybrid neurosurgeons who conduct concurrently with open surgery and neurointerventional procedure : 48 procedures for ruptured aneurysm; five unruptured aneurysm; six ruptured arteriovenous malformation (AVM); two rupture dural arteriovenous fistula; 103, ischemic stroke; and 27, intracranial hemorrhage (ICH). After the review of all cases, we selected cases which were treated using hybrid technique. The procedures were categorized into three subcategories : 1) combined endovascular and surgical procedure, 2) complementary rescue procedure of intervention and surgery, and 3) frameless stereotaxic operation.

\section{Organization and equipment of hybrid operating room}

A ceiling-mounted monoplane DSA system (Allura Xper ${ }^{\circledR}$ 20FD; Philips Medical Systems, Best, the Netherlands) was installed in one of our ORs. The system consists of a 3DRA, Carm mounted CBCT, and virtual guiding software (Xper CT
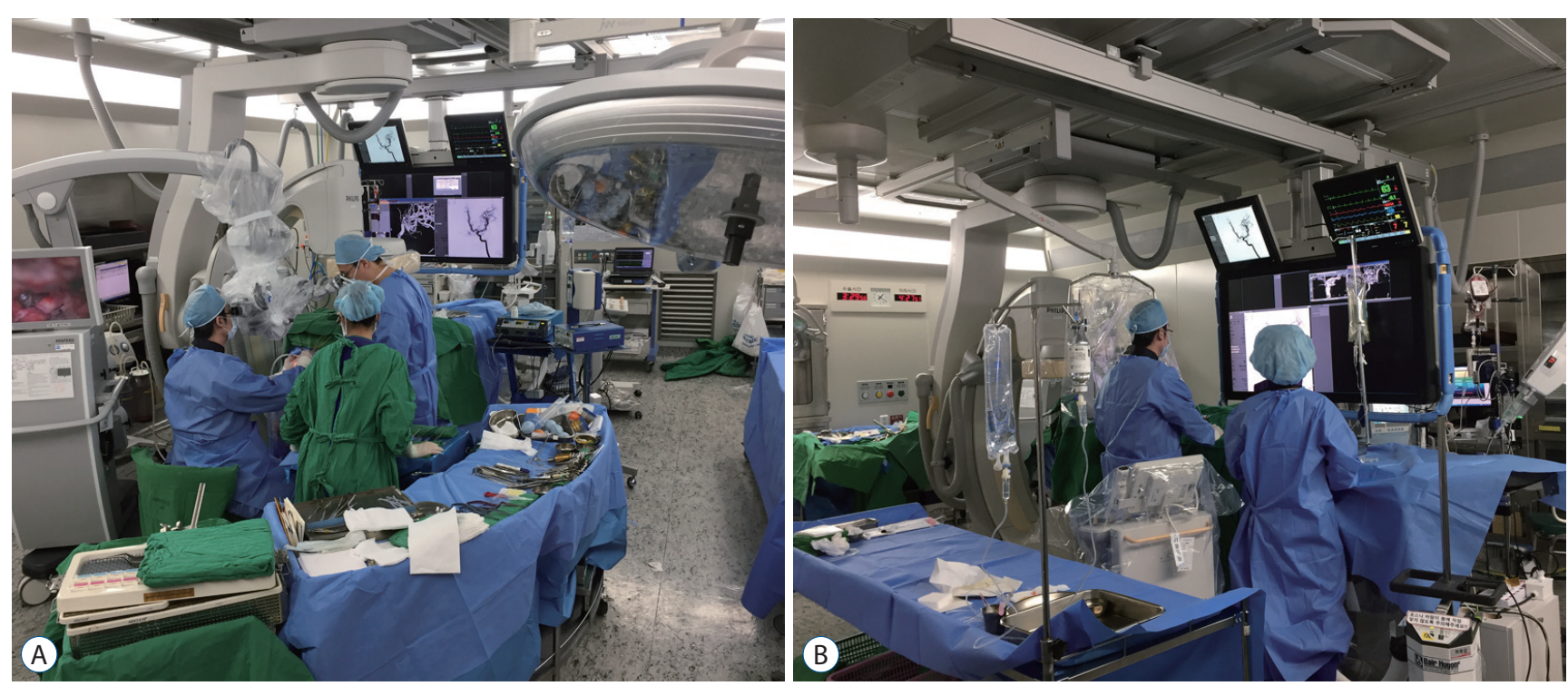

Fig. 1. A: Configuration of the hybrid operating room during the cranial operation. B: Positioning of the fluoroscopic C-arm during the endovascular procedure. 
and XperGuide System; Philips Medical Systems). The hybrid OR was also equipped with an X-ray transparent surgical table (MAGNUS OR Table System; Maquet GmbH, Rastatt, Germany) and a radiolucent skull clamp, halo ring, and horseshoe headrest $\left(\mathrm{DORO}^{\circledR}\right.$ Radiolucent Headrest System; Pro Med Instruments, GmbH, Germany). This system allows intraoperative angiography with 3DRA and 320 or 640 projections around $240^{\circ}$ with a rotational speed of 30 or $55^{\circ}$ per second. Both intraoperative angiography with 3DRA and CBCT can be performed any time during surgery without moving the patient to another table or position. If the surgical table is tilted at any degree and direction during surgery, the surgical table should be returned to neutral position to perform 3DRA and CBCT. Radiolucent headrest system is usually maintained during intraoperative angiography with 3DRA and CBCT. During cranial surgery, the C-arm falls to the caudal side. In addition, when performing angiography, intervention, or CBCT, the C-arm moves to the cranial side (Fig. 1).

\section{RESULTS}

Forty-nine of 191 procedures were performed using hybrid techniques in a single session without any problems (Table 1).

\section{Combined endovascular and surgical procedure}

Four cases of blood blister-like aneurysm (BBA) and one ruptured aneurysm of the posterior inferior cerebellar artery (PICA) were treated by one-off combined bypass surgery and endovascular trapping of the pathologic segment (Fig. 2). In emergency situations, the balloon occlusion test was not performed to evaluate the collateral flow. In cases of blister aneurysm, the carotid compression test was performed to reveal angiographic collateral flow. In all cases of BBA, poor collateral flow was identified. In all cases, bypass surgery was performed first, and subsequently, coil embolization was performed. During interventional procedure, craniotomy was left open in cases of anterior circulation lesion. After coil embolization, further dissection was done to identify the lesions. In a case of posterior circulation lesion, craniotomy was closed before coil embolization because the patient's position needed to change from prone to supine. All procedures were done without any complication.

Eight cases of ruptured aneurysm with $\mathrm{ICH}$ were treated by subtotal coil embolization and subsequent clip ligation (Fig. 3), of which five were middle cerebral artery bifurcation aneurysm; two posterior communicating artery aneurysm; and one anterior communicating artery aneurysm. The coils were visible through the aneurysm dome, and no occurrence of intraoperative aneurysm rupture was reported in all cases. All aneurysms were eliminated without any procedural complications. Systemic heparinization was not performed for endovascular procedures in all cases. Continuous irrigation was maintained using saline mixed with heparin via a guiding

Table 1. Details of procedures

\begin{tabular}{|c|c|c|c|c|}
\hline & No. & Procedure 1 & Procedure 2 & Procedure 3 \\
\hline \multicolumn{5}{|c|}{ Combined endovascular and surgical procedure } \\
\hline Ruptured AVM & 6 & Preoperative embolization & Surgical resection & ICH removal \\
\hline Blister aneurysm & 4 & High-flow bypass & Embo trapping & \\
\hline Ruptured PICA An. & 1 & OA-PICA bypass & Embo trapping & \\
\hline Ruptured An. with ICH & 8 & Partial An. embolization & Clipping & ICH removal \\
\hline \multicolumn{5}{|l|}{ Rescue procedure } \\
\hline Premature rupture of $A n$. & 2 & Sylvian dissection & An. embolization & Clipping \\
\hline Complicated IA thrombecomy & 1 & IA thrombectomy & Open thrombectomy & ECIC bypass \\
\hline \multicolumn{5}{|l|}{ Frameless stereotaxic operation } \\
\hline Spontaneous ICH & 20 & Cone beam $\mathrm{CT}$ & ICH aspiration & \\
\hline Ruptured An. with ICH & 5 & An. clipping & Cone beam $\mathrm{CT}$ & ICH aspiration \\
\hline Ruptured dAVF with ICH & 2 & dAVF embolization & Cone beam CT & $\mathrm{ICH}$ aspiration \\
\hline
\end{tabular}

AVM : arteriovenous malformation, ICH : intracranial hemorrhage, Embo : embolization, PICA : posterior inferior cerebellar artery, An. : aneurysm, OA : occipital artery, IA : intra-arterial, ECIC : external carotid-internal carotid, CT : computed tomography, dAVF : dural arteriovenous malformation 
catheter and microcatheter. There was no ischemic complication after the procedures.

Six patients of ruptured cerebral AVM underwent combined endovascular and surgical procedures in the hybrid OR. In all cases, preoperative embolization was done first. Subsequent surgical procedures were performed under indocyanine green video angiography (ICG-VA) guidance. In all cases, ICG-VA could not reveal residual nidus. In one (16.7\%) of six procedures, however, residual nidus was found by intraoperative angiography. This residual lesion was resected in the same session and second angiography confirmed completion of resections.

\section{Illustrative case 1}

A 64-year-old female patient presented with semicomatose mentality. Brain computed tomography (CT) showed diffuse subarachnoid hemorrhage (SAH) with intraventricular hemorrhage and hydrocephalus (Fig. 2A). On DSA, the aneurysm at the proximal segment of the left PICA was identified. It had a wide neck with triangular shaped dome. It also associated with parent artery stenosis at the juxtadistal to the aneurysm (Fig. 2B). We decided that the lesion was not suitable for endovascular treatment, and clipping maintaining patency of the parent artery also seemed to be difficult due to geometry of the aneurysm. Under the general anesthesia, the patient placed in the prone position. We performed extraventricular drainage catheter insertion at the right parieto-occipital point, and occipital artery (OA)-PICA bypass through the left suboccipital craniotomy (Fig. 2C). After the patient placed supine position, subsequent endovascular trapping underwent to occlude the aneurysm including parent artery (Fig. 2D). Intraoperative angiogram revealed complete occlusion of the aneurysm with
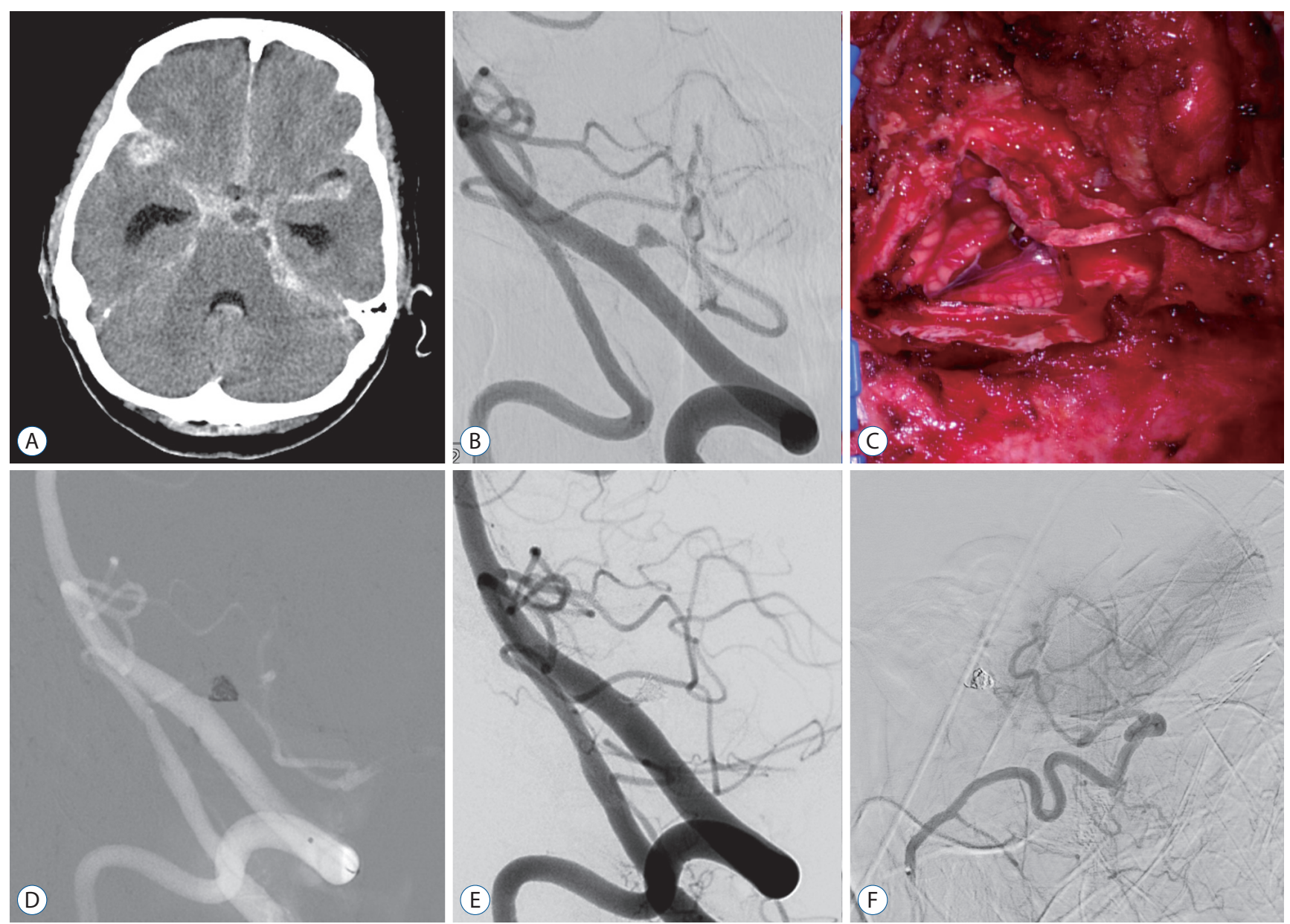

Fig. 2. Brain computed tomography and digital subtraction angiography showed a dissecting aneurysm at the proximal segment of the left posterior inferior cerebellar artery (PICA) with diffuse subarachnoid hemorrhage (A and B). C : Occipital artery (OA)-PICA bypass was performed first. D : Subsequent coil embolization was performed. Complete control intraoperative angiogram showed complete occlusion of the aneurysm with patent OA-PICA bypass (E and F). 
patent OA-PICA bypass (Fig. 2E and F). The patient fully recovered without any neurologic deficits.

\section{Illustrative case 2}

A 53-year-old female patient presented with severe headache.
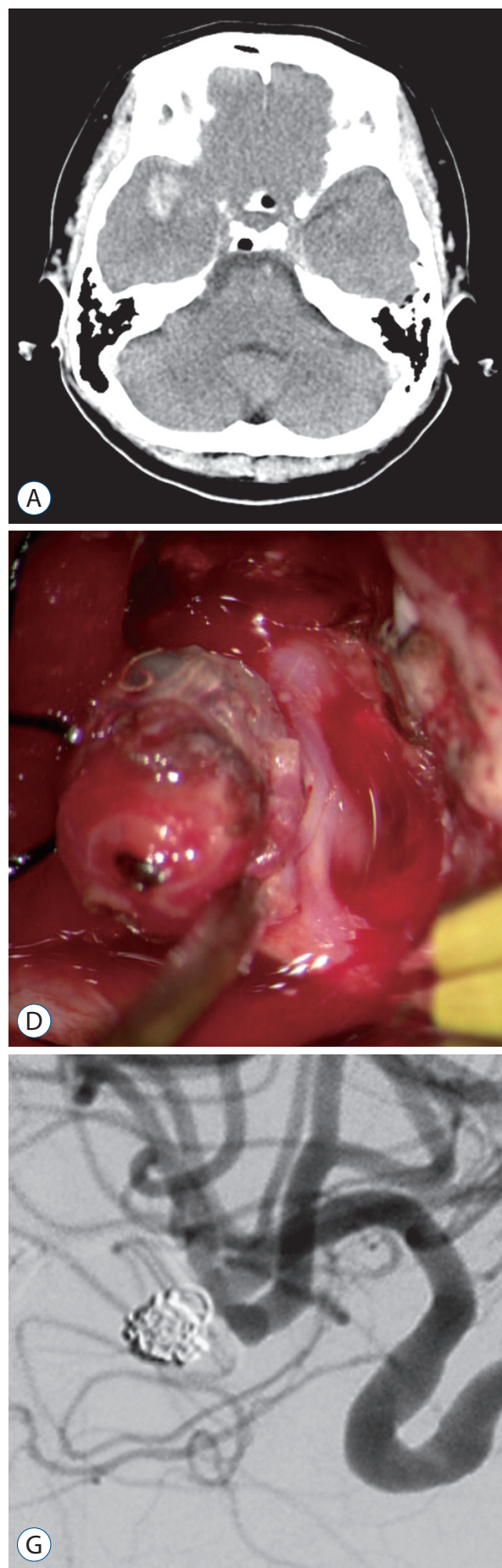
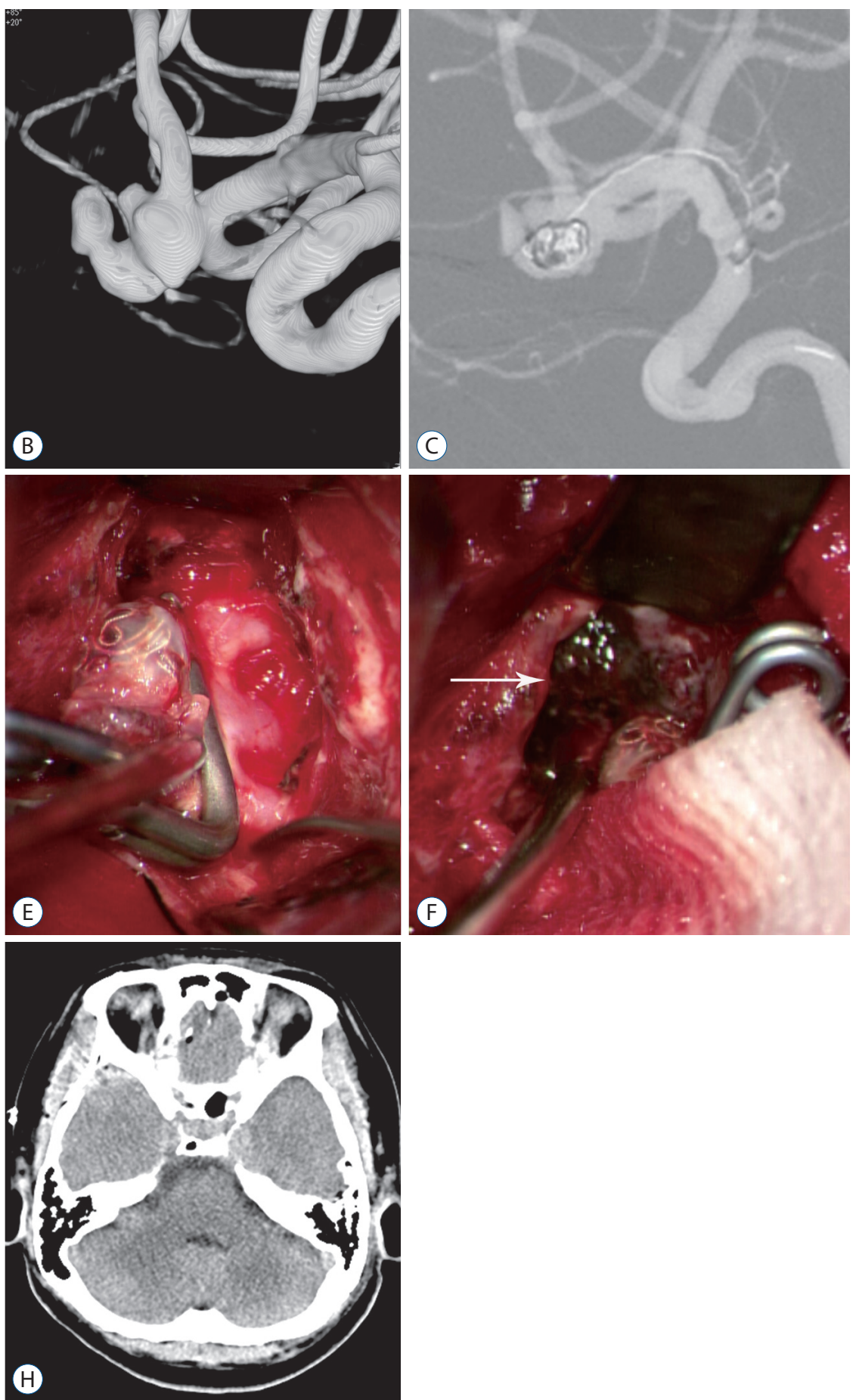

Fig. 3. A : Noncontrast brain computed tomography (CT) showed diffuse subarachnoid hemorrhage with a left temporal hemorrhage. B : 3-dimensional rotational angiogram revealed complex ruptured aneurysm of the left middle cerebral artery bifurcation. C: Subtotal coil embolization was done to protect ruptured point. Neck of the aneurysm was preserved. D : Partially coiled aneurysm was exposed without pre-mature rupture. E : Clip application was done with preservation of the parent artery. F : Temporal hemorrhage was exposed nearby coiled segment of the aneurysm (arrow), and it was removed under the microscope. G : Intraoperative angiography showed complete occlusion of the aneurysm. $\mathrm{H}$ : Postoperative brain CT revealed complete removal of the temporal hemorrhage. 
Brain CT scan showed diffuse SAH with the right temporal hemorrhage (Fig. 3A). DSA demonstrated a complex aneurysm at the right middle cerebral artery (MCA) bifurcation (Fig. 3B). To protect pre-mature rupture during sylvian dissection, bareplatinum coils were embolized in the suspected rupture point (Fig. 3C). After that, aneurysm exposure was done. We could expose the whole aneurysm without premature rupture (Fig. 3D). Even though it was a multi-lobulated, wide-necked aneurysm, we could achieve complete aneurysm occlusion without residual neck preserving parent artery (Fig. 3E). Temporal hemorrhage was exposed during aneurysm dissection, and we could surgically remove under the microscopic field after clip application (Fig. 3F). Intraoperative angiogram revealed complete occlusion of aneurysm, and postoperative brain CT showed complete removal of the temporal hemorrhage (Fig. 3G and H).
The patient was discharged without any neurologic deficits.

\section{Complementary rescue procedure of interven- tion and surgery}

In two (4.5\%) pre-mature rupture during clipping of the 44 ruptured aneurysm cases, they were secured by endovascular coil embolization. Intraoperative aneurysm rupture occurred early in sylvian dissection before exposing the aneurysm and parent artery in two cases. When intraoperative aneurysm rupture occurred, the double suction technique was applied to identify the bleeding point. However, bleeding point could not be clarified because the sylvian dissection was not complete. Moreover, the aneurysm and parent artery were not exposed, and bleeding control could not be done with temporary clip or tandem clip application. Under the suction, a cotton pad
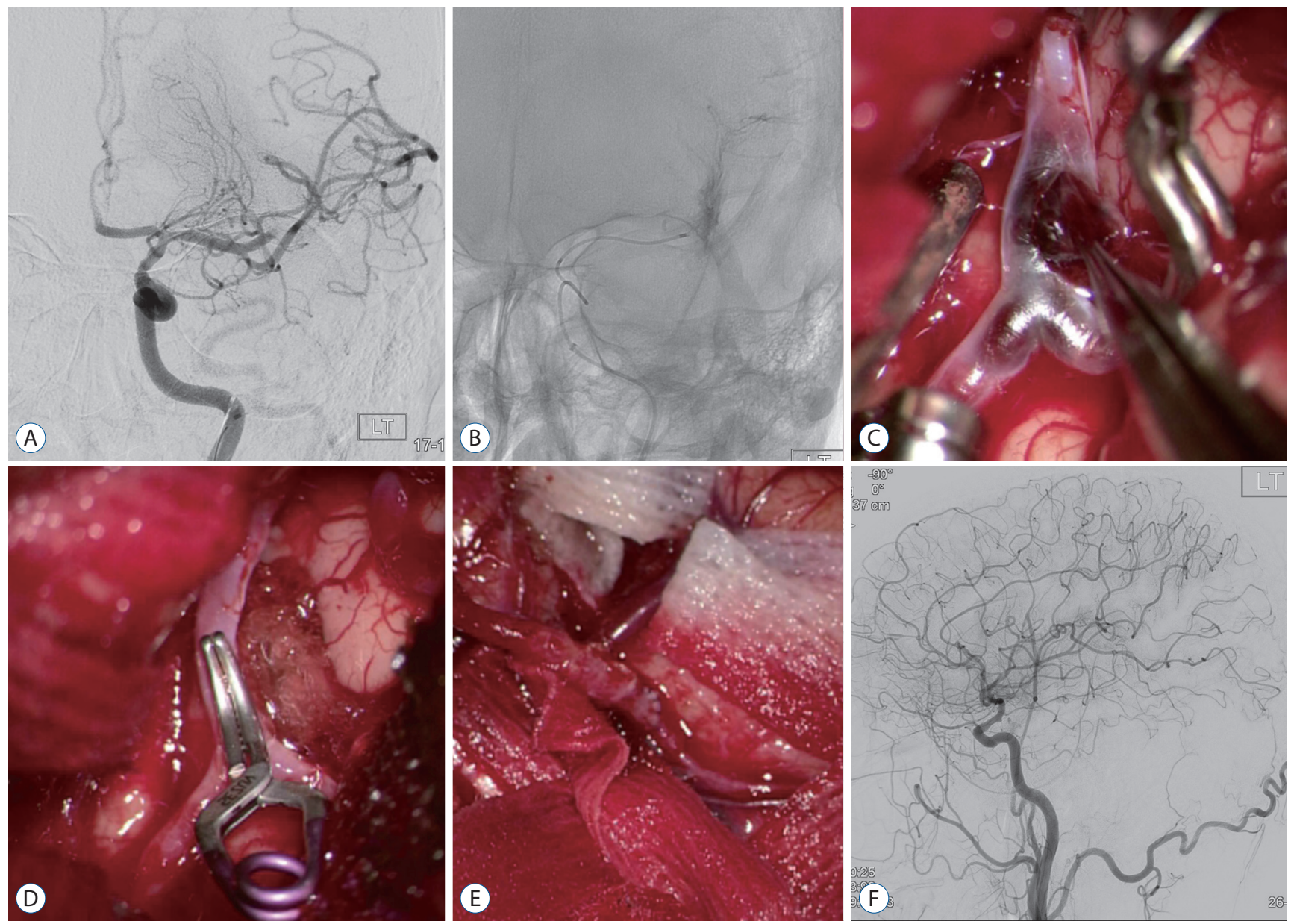

Fig. 4. A : The left internal carotid angiogram revealed occlusion of middle cerebral artery (MCA) superior trunk. B: Superselective angiogram via microcatheter showed contrast leakage. C : Surgical embolectomy was performed. D : Arteriotomy site was closed using right-angled mini-clip. E : Superficial temporal arteryMCA bypass was performed for central artery occlusion after surgical embolectomy. Complete surgical recanalization was achieved 367 minutes after the last normal time. F: Complete control angiogram showed complete recanalization of the left MCA. 
was compressed against the bleeding point beneath the suction until bleeding stopped. Immediately after bleeding control was achieved, we were able to switch to endovascular procedure remaining the craniotomy site open. After coil embolization, microdissection was performed to expose the aneurysm, and clipping was performed if necessary.

In one $(0.8 \%)$ complicated case of the 103 intra-arterial (IA) thrombectomy procedures, of which further interventional procedures were impossible, emergency surgical embolectomy with bypass surgery was performed. Even though intravenous (IV) tissue plasminogen activator (tPA) was prescribed, no procedural-related hemorrhagic complications were reported (Fig. 4). The patient presented a National Institute of Health Stroke Scale (NIHSS) score of 22, and the NIHSS score at dis- charge was 2.

\section{Illustrative case 3}

A 55-year-old male patient presented with aphasia and right side weakness. Initial symptoms developed 30-minute before admission. Brain CT showed no abnormal findings, however, CT perfusion revealed transit time delay at the left MCA territory. Left internal carotid angiogram demonstrated occlusion of proximal segment of MCA superior trunk (Fig. 4A). Initial NIHSS score was 20, and immediate endovascular thrombectomy underwent under the local anesthesia. After the navigation of occlusion segment using microcatheter (Prowler Select PLUS; Codman Neurovascular, Miami Lakes, FL, USA) and microwire (Synchro 14; Stryker, Fremont, CA, USA), superse-
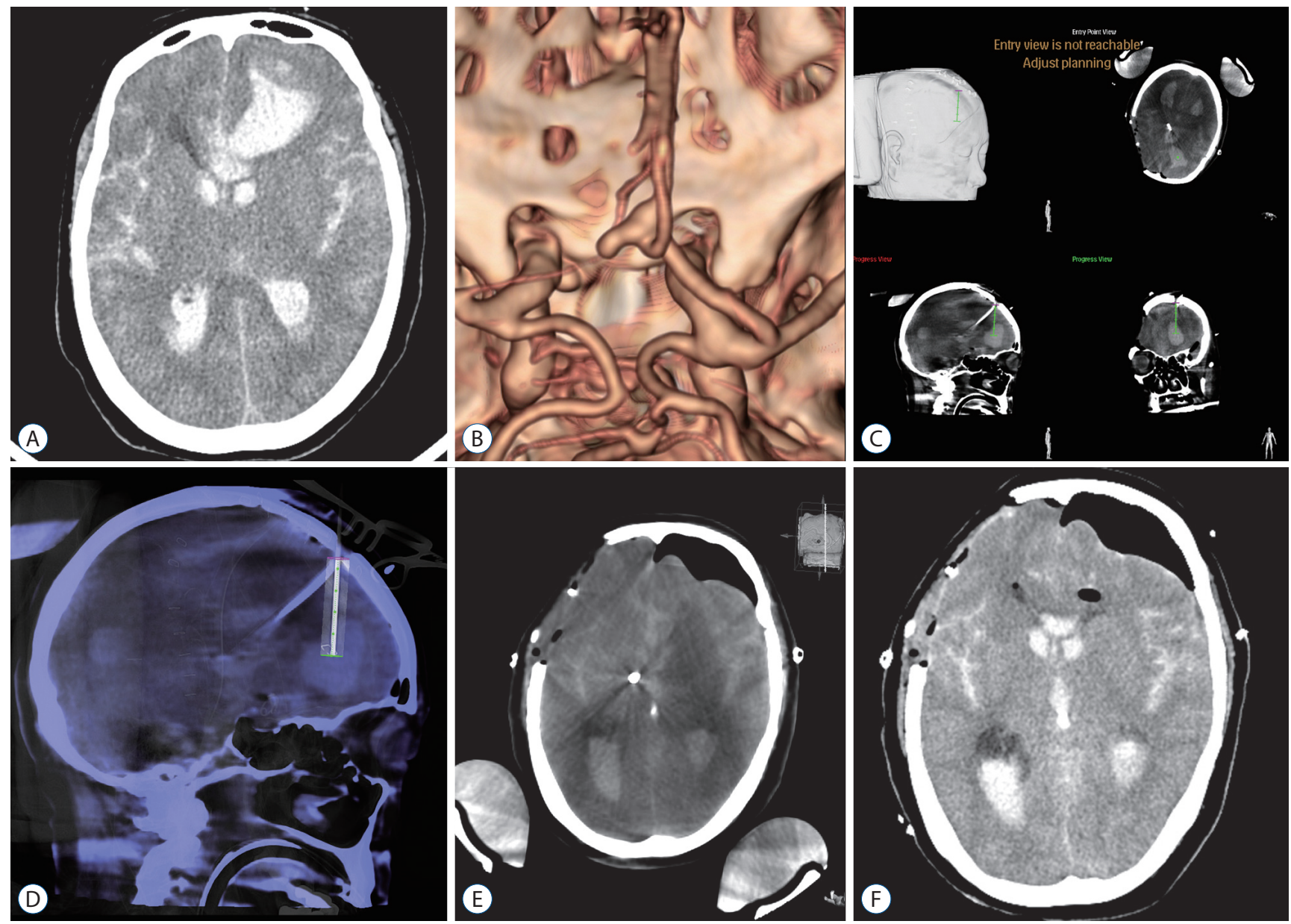

Fig. 5. A and B : Brain computed tomography (CT) and CT angiography showed an aneurysm at the anterior communicating artery with diffuse subarachnoid hemorrhage accompanied by intracranial hemorrhage $(\mathrm{ICH})$ at the left frontal lobe. $\mathrm{C}$ : After clip application through the right pterional approach, ICH was aspirated under the guidance of XperGuide ${ }^{\varpi}$ system (Philips Medical Systems, Best, the Netherlands) via the left Kocher's point. D: During progression of the needle, trajectory was shown on the fluoroscopic image as central needle path with surrounding cylinder of 1-cm safety margin. E : Post-aspiration cone-beam CT showed near-complete removal of ICH. F : Postoperative brain CT showed near-complete removal of the frontal ICH. 
lective angiography was done. It showed contrast leakage into the subarachnoid space (Fig. 4B). The NIHSS score was worsen to 22. We decided immediate conversion to open surgery for surgical embolectomy. After induction of general anesthesia, the left pterional approach was done. During sylvian dissection, rupture point was identified, and it could be controlled using bipolar cautery. Further dissection identified occlusion segment of MCA. Using a microscissor, arteriotomy was done at the mid-segment of thrombus. Thrombus was retrieved using Jeweller's forceps, and arteriotomy site was closed using right-angled mini-clip (Fig. 4C and D). Intraoperative angiogram demonstrated an occlusion of central artery, and emergent superficial temporal artery (STA)-MCA bypass was done (Fig. 4E). Complete control angiography showed complete recanalization of the left MCA (Fig. 4F). Complete surgical recanalization was achieved 367 minutes after the last normal time. There were no surgical complications. At discharge, patient's NIHSS score was 2.

\section{Frameless stereotaxic operation}

In 27 cases of ICH, frameless stereotaxic hematoma aspiration was performed using XperGuide system. Of the 27 cases, 20 had spontaneous ICH; five aneurysm-related ICH; and two ICH from a ruptured dural AVF. All cases required more than one aspiration. Immediately after the first aspiration, XperCT was performed to evaluate residual hematoma and re-planning for further session of aspiration, if necessary. Therefore, we can achieve near-complete removal of ICH. In two (10\%) of the 20 cases of spontaneous ICH, decompressive craniectomy was performed due to cerebral edema (Fig. 5).

\section{Illustrative case 4}

A 65-year-old female patient presented with stuporous mentality. Brain CT and CT angiography showed diffuse SAH with large left frontal ICH (Fig. 5A and B), and anterior communicating artery (Acom) aneurysm. Due to the right A1 dominancy with aplastic left A1, microsurgery was performed through the right pterional approach. After clip application for the Acom aneurysm, ICH was aspirated via burr-hole at the left Kocher's point under the guidance of XperGuide ${ }^{\circledR}$ system (Philips Medical Systems) (Fig. 5C and D). Without additional craniotomy, we could achieve near-complete removal of the left frontal ICH by two-session of aspiration (Fig. $5 \mathrm{E}$ and $\mathrm{F}$ ). The patient was recovered with mild cognitive im- pairment (modified Rankin Scale score 1).

\section{DISCUSSION}

We observed the feasibility, safety, and impact of hybrid OR on the treatment of various cerebrovascular diseases. For complex intracranial aneurysms, combined microsurgical and endovascular technique could provide relatively simple and safe treatment strategies ${ }^{3}$. Direct clipping of BBA usually has a relatively high rate of intraoperative aneurysm rupture during acute phase surgery ${ }^{21,222}$. Although the endovascular procedure is presented as an alternative, there is a relatively high probability of rupture during coil embolization and recurrence even after treatment ${ }^{22}$. Thus, trapping of ICA involving BBA with/ without bypass surgery is considered to be a relatively safe and complete procedure to treat $\mathrm{BBA}^{8,9)}$. In microsurgical trapping, anterior clinoidectomy is often necessary, and sensitive lesions should be touched. In contrast, endovascular trapping can be performed safely and conveniently compared to microsurgical trapping. However, an optimal treatment method for BBAs had not been defined. Various surgical and endovascular strategies for BBAs have been suggested, and each method has usually been applied to each case. A hybrid procedure for BBAs that we present in this study is another option when both methods are not appropriate to apply. In the case of PICA aneurysm, extensive bone work was not required to expose the lesion. Once bypass flow has been guaranteed, endovascular trapping of aneurysm could simplify the operation from a far lateral approach with vertebral artery exposure and condylectomy. In these treatments, bypass occlusion could be a limitation of two-session combined treatment, and singlesession combined treatment is a better option to avoid bypass occlusion $^{15)}$.

Treatment of ruptured aneurysms with ICH is also challenging. Microsurgical clipping of ruptured aneurysms associated with ICH often involves intraoperative aneurysm rupture during dissection, and exposing wide-necked or large-sized lesions is often difficult due to brain swelling or $\mathrm{ICH}^{18)}$. In terms of endovascular procedures, early rebleeding often occurs after coil embolization of ruptured aneurysm with adjacent $\mathrm{ICH}^{2,10,17)}$.

For wide-necked lesions, complete occlusion may be difficult using coil embolization ${ }^{4,25}$. In the case of these lesions, 
preventing pre-mature rupture during dissection, we performed incomplete coil embolization to protect the ruptured point of the aneurysm. All eight incompletely coil-embolized aneurysms could be dissected without intraoperative aneurysm rupture and completely obliterated using clip. After incomplete coil embolization, in particular, microsurgical dissection around the aneurysm could be performed as in case of unruptured aneurysm. Consequently, we could expose the entire aneurysm with the parent artery, and exquisite clip application can be performed in cases of complex aneurysms.

The use of combined surgical and endovascular treatment of AVMs has been applied; however, most of the combined procedures were usually performed in separate sessions. We highlight the value of hybrid OR in allowing neurosurgeons to treat ruptured AVMs effectively and safely with combined endovascular and surgical techniques simultaneously in emergency situations. In addition, hybrid OR offers intraoperative angiography with 3DRA and CBCT, which further enhances surgical results and patient safety during the procedure. Surgical outcomes were evaluated by intraoperative angiography with 3DRA, and complete AVM removal could be achieved in the same surgical session, if necessary.

Intraoperative aneurysm rupture during aneurysm surgery is a critical event and can be potentially devastating ${ }^{3,8)}$. In particular, if an intraoperative aneurysm rupture occurs when an aneurysm and surrounding arteries are not exposed, clipping is not possible, and further dissection may be difficult. In a hybrid OR, emergency coil embolization could be performed by femoral puncture immediately after bleeding control via vigorous suction and compression. This allowed further dissection to confirm the aneurysm and clipping if necessary. All of the two patients described above showed good clinical outcomes.

In a complicated IA thrombectomy case, hybrid OR allowed immediate conversion to open surgery. Bleeding focus was identified, and it was controlled using bipolar cautery. In addition, open thrombectomy and STA-MCA bypass were performed for the occlusion segment of intracranial arteries. Since the half-life of tPA is very short, surgical treatment can be safely performed even after IV infusion of tPA. The potentially hazardous situation could be overcome through an immediate switch to another treatment strategy in hybrid OR, and we could achieve relatively good outcomes in complicated cases.
CBCT is a technology that allows 3D image acquisition via a C-arm fluoroscopy system, and it has good bone and soft tissue resolution ${ }^{20,24)}$. Fully equipped high-end DSA also provides a real-time 3D fluoroscopy guidance system for needle interventions with dedicated planning software. This system has been used in several areas where needle intervention is applied and proven to provide high accuracy despite the respiration motion ${ }^{1,712,16)}$. We applied XperGuide system to ICH and could achieve near-total removal of ICH without any procedural complications. The brain was not interfered with the respiration of the patient, allowing more precise targeting. After the first aspiration, new targeting was possible reflecting the changed intracranial structure via repeated CBCTs, and it provides more precise therapeutic tools for ICH aspiration compared with conventional neuronavigation systems. Aneurysm-related ICHs inaccessible from the surgical field for clipping could be removed without additional craniotomy via the XperGuide system in a single-session procedure without any additional imaging study for neuronavigation. Furthermore, when navigation surgery is needed urgently without pre-operative images for registration, frameless stereotaxic surgery could be provided in hybrid OR.

Hybrid OR would not provide a completely different treatment strategy for the treatment of cerebrovascular disease. Basically, using the advantages of surgical treatment and intervention in one place, we could overcome limitations of each treatment modality. Especially, hybrid OR could suggest new strategies to cope flexibly with complex lesions or unexpected situations. Hybrid OR could provide part of these feasible, modified strategies, and we believe that it could contribute to more precise and patient-safe alternatives using hybrid OR for cerebrovascular disease.

\section{Drawbacks of hybrid operating room}

The hybrid OR fully equipped a high-end DSA system provides useful strategies to treat cerebrovascular disease; however, there are still some drawbacks to be overcome. In terms of radiation dose for the surgeon, nurse, and anesthesiologist, it must be monitored and shielded. Although recently introduced radiation dose reduction fluoroscopic technology showed a decrease in radiation dose to both patient and operator, lifetime radiation dose accumulation would be associated with hazardous biologic effect. To minimize intraoperative radiation dose, appropriate lead shields, such as lead aprons, 
lead goggles, and thyroid protectors, should be provided during regular fluoroscopic procedures. In addition, all staff should be out of OR during 3DRA or CBCT acquisition. Patient's position is usually limited to basic position. Even though newly designed hybrid operating table has been introduced, range of table movement is also restricted ${ }^{19)}$. In case of a posterior circulation lesion requiring surgical procedures such as bypass, the patient's position should be converted from prone to supine after surgery to perform subsequent endovascular procedures. During 3DRA or CBCT, the operative table should be returned to neutral position. If additional surgical procedure is needed, the patient's position should be appropriately re-set for surgery after 3DRA or CBCT. Sterility is also a concern in hybrid OR. During intraoperative angiography or combined surgical and endovascular procedures, surgical field is usually remained open. Because $\mathrm{C}$-arm is moved around the opened surgical field, C-arm should be appropriately covered using sterile covers. At the same time, the opened surgical field also should be covered using sterile drapes. To minimize contamination of surgical and endovascular field during conversion to each procedure, cranial and inguinal drape was prepared simultaneously before the procedure. In our preliminary experience, no postoperative infections occurred. For neurointerventional use, the biplane angiographic system still remains the gold standard. Therefore, most scheduled neurointerventional procedures have been performed in a biplane angiography suite. Finally, there must be close cooperation between neurosurgeons, neurointerventionists, anesthesiologists, and radiologic technologists, as well as manager of the operating rooms and nurses. In particular, interdisciplinary approaches and collaboration between physicians performing open surgery and endovascular procedures would provide better treatment strategies for patients.

\section{Limitations}

Aside from the general limitations of the retrospective, single-center study, there is another limitation in this study. Due to the small number of cases appertaining to each subcategory, comparison with the clinical outcomes obtained by conventional strategies and hybrid procedures of each subcategory was not feasible. Thus, usefulness of hybrid procedure for cerebrovascular diseases needs further confirmation in a large prospective study.

\section{CONCLUSION}

Hybrid OR with a fully equipped DSA system could provide safe and precise treatment in the field of neurosurgery. A combined endovascular and surgical approach performed in a hybrid OR would provide new strategies for complex cerebrovascular diseases, which are more protected and simplified in comparison with surgical or endovascular approach alone. In complicated surgical or endovascular procedures, quick conversion to backup surgery or endovascular procedure in hybrid OR could provide safety and overcome hazardous complications. In addition, СBCT would be useful as a tool of intraoperative CT and frameless stereotaxic surgery without preoperative images.

\section{CONFLICTS OF INTEREST}

No potential conflict of interest relevant to this article was reported.

\section{INFORMED CONSENT}

This type of study does not require informed consent.

\section{- Acknowledgements}

This research was supported by Hallym University Research Fund (HURF-2016-32).

\section{References}

1. Braak SJ, van Strijen MJ, van Leersum M, van Es HW, van Heesewijk JP : Real-time 3D fluoroscopy guidance during needle interventions: technique, accuracy, and feasibility. AJR Am J Roentgenol 194 : W445W451, 2010

2. Chang SH, Shin HS, Lee SH, Koh HC, Koh JS : Rebleeding of ruptured intracranial aneurysms in the immediate postoperative period after coil embolization. J Cerebrovasc Endovasc Neurosurg 17 : 209-216, 2015

3. Chen SF, Kato Y, Kumar A, Tan GW, Oguri D, Oda J, et al. : Intraoperative rupture in the surgical treatment of patients with intracranial aneurysms. J Clin Neurosci 34 : 63-69, 2016

4. Cho WS, Kim JE, Park SQ, Ko JK, Kim DW, Park JC, et al. : Korean clinical practice guidelines for aneurysmal subarachnoid hemorrhage. J Korean 
Neurosurg Soc 61 : 127-166, 2018

5. Fandino J, Taussky P, Marbacher S, Muroi C, Diepers M, Fathi AR, et al. : The concept of a hybrid operating room: applications in cerebrovascular surgery. Acta Neurochir Suppl 115 : 113-117, 2013

6. Fong YW, Hsu SK, Huang CT, Hsieh CT, Chen MH, Huang JS, et al. : Impact of intraoperative 3-dimensional volume-rendering rotational angiography on clip repositioning rates in aneurysmal surgery. World Neurosurg 114 : e573-e580, 2018

7. Gautschi OP, Smoll NR, Kotowski M, Schatlo B, Tosic M, Stimec B, et al. : Non-assisted versus neuro-navigated and XperCT-guided external ventricular catheter placement: a comparative cadaver study. Acta Neurochir (Wien) 156 : 777-785; discussion 785, 2014

8. Hsu CE, Lin TK, Lee MH, Lee ST, Chang CN, Lin CL, et al. : The impact of surgical experience on major intraoperative aneurysm rupture and their consequences on outcome: a multivariate analysis of 538 microsurgical clipping cases. PLoS One 11 : e0151805, 2016

9. Jadhav AP, Ribo M, Grandhi R, Linares G, Aghaebrahim A, Jovin TG, et al. : Transcervical access in acute ischemic stroke. J Neurointerv Surg $6: 652-657,2014$

10. Jartti $P$, Isokangas JM, Karttunen $A$, Jartti $A$, Haapea M, Koskelainen $T$, et al. : Early rebleeding after coiling of ruptured intracranial aneurysms. Acta Radiol 51 : 1043-1049, 2014

11. Kawamura Y, Sayama T, Maehara N, Nishimura A, lihara K : Ruptured aneurysm of an aberrant internal carotid artery successfully treated with simultaneous intervention and surgery in a hybrid operating room.

World Neurosurg 102 : 695.e1-695.e5, 2017

12. Lee SM, Park CM, Lee KH, Bahn YE, Kim Jl, Goo JM : C-arm cone-beam CT-guided percutaneous transthoracic needle biopsy of lung nodules: clinical experience in 1108 patients. Radiology 271 : 291-300, 2014

13. Mori R, Yuki I, Kajiwara I, Nonaka Y, Ishibashi T, Karagiozov K, et al. : Hybrid operating room for combined neuroendovascular and endoscopic treatment of ruptured cerebral aneurysms with intraventricular hemorrhage. World Neurosurg 89 : 727.e9-727.e12, 2016

14. Murayama $Y$, Irie $K$, Saguchi $T$, Ishibashi $T$, Ebara M, Nagashima $H$, et al. : Robotic digital subtraction angiography systems within the hybrid operating room. Neurosurgery 68 : 1427-1432; discussion 1433, 2011

15. Murayama Y, Arakawa H, Ishibashi T, Kawamura D, Ebara M, Irie K, et al. : Combined surgical and endovascular treatment of complex cerebro- vascular diseases in the hybrid operating room. J Neurointerv Surg 5 : 489-493, 2013

16. Nesbit GM, Nesbit EG, Hamilton BE : Integrated cone-beam CT and fluoroscopic navigation in treatment of head and neck vascular malformations and tumors. J Neurointerv Surg 3 : 186-190, 2011

17. Ohta T, Murao K, Miyake K, Takemoto K, Nakazawa K : Risk factors for early hemorrhagic complications after endovascular coiling of ruptured intracranial aneurysms. AJNR Am J Neuroradiol 35 : 2136-2139, 2014

18. Park J, Son W, Park KS, Kang DH, Shin IH : Intraoperative premature rupture of middle cerebral artery aneurysms: risk factors and sphenoid ridge proximation sign. J Neurosurg 125 : 1235-1241, 2016

19. Park JH, Mun JH, Shin DS, Kim BT : Novel hybrid operating table for neuroendovascular treatment. Turk Neurosurg, 2017 [Epub ahead of print]

20. Ritter D, Orman J, Schmidgunst C, Graumann R : 3D soft tissue imaging with a mobile C-arm. Comput Med Imaging Graph 31 : 91-102, 2007

21. Satoh A, Sugiyama T, Hongo K, Kakizawa Y, Ishihara S, Matsutani M : Nationwide surveillance of ic anterior (or dorsal) wall aneurysm: with special reference to its dissecting nature. Acta Neurochir Suppl 103 : 51-55, 2008

22. Shah SS, Gersey ZC, Nuh M, Ghonim HT, Elhammady MS, Peterson EC : Microsurgical versus endovascular interventions for blood-blister aneurysms of the internal carotid artery: systematic review of literature and meta-analysis on safety and efficacy. J Neurosurg 127 : 1361-1373, 2017

23. Shen SC, Tsuei YS, Chen WH, Shen CC : Hybrid surgery for dural arteriovenous fistula in the neurosurgical hybrid operating suite. J Neurointerv Surg $7:$ e6, 2015

24. Siewerdsen JH, Moseley DJ, Burch S, Bisland SK, Bogaards A, Wilson BC, et al. : Volume CT with a flat-panel detector on a mobile, isocentric Carm: pre-clinical investigation in guidance of minimally invasive surgery. Med Phys 32 : 241-254, 2005

25. White AC, Roark CD, Case DE, Kumpe DA, Seinfeld I : Factors associated with rerupture of intracranial aneurysms after endovascular treatment: a retrospective review of 11years experience at a single institution and review of the literature. J Clin Neurosci 44 : 53-62, 2017 